\title{
Bayesian methods for hierarchical distance sampling models
}

\author{
C. S. Oedekoven ${ }^{1, *}$, S. T. Buckland ${ }^{1}$, M. L. Mackenzie ${ }^{1}$, \\ R. King ${ }^{1}$, K. O. Evans ${ }^{2}$, and L. W. Burger, Jr. ${ }^{2}$ \\ ${ }^{1}$ Centre for Research into Ecological and Environmental Modelling, \\ School of Mathematics and Statistics, \\ University of St Andrews, St Andrews, KY16 9LZ, UK \\ ${ }^{2}$ Department of Wildlife, Fisheries \& Aquaculture, Mississippi State University, \\ Box 9690, Mississippi State, MS 39762, USA \\ *email: cornelia@mcs.st-and.ac.uk
}

\begin{abstract}
The few distance sampling studies that use Bayesian methods typically consider only line transect sampling with a half-normal detection function. We present a Bayesian approach to analyse distance sampling data applicable to line and point transects, exact and interval distance data and any detection function possibly including covariates affecting detection probabilities. We use an integrated likelihood which combines the detection and count models. For the latter, counts are related to covariates in a log-linear mixed effect Poisson model which accommodates correlated counts. We use a Metropolis-Hastings algorithm for updating parameters and a reversible jump algorithm to include model selection for both the detection function and count models. The approach is applied to a large-scale experimental design study of northern bobwhite coveys where the interest was to assess the effect of establishing herbaceous buffers around agricultural fields in several states in the US on bird densities. Results were compared with those from an existing maximum likelihood approach that analyses the detection and count models in two stages. Both methods revealed an increase of covey densities on buffered fields. Our approach gave estimates with higher precision even though it does not condition on a known detection function for the count
\end{abstract}


model.

Keywords: designed experiments, hazard-rate detection function, heterogeneity in detection probabilities, Metropolis-Hastings update, point transect sampling, RJMCMC.

\section{Introduction}

Bayesian methods are becoming increasingly popular for modelling wildlife populations and abundances (e.g. Buckland et al., 2000, Marcot et al., 2001; Durban and Elston, 2005; Schmidt et al., 2009; King et al., 2010). However, few distance sampling studies have taken a Bayesian approach. Karunamuni and Quinn (1995) developed a Bayes estimator for $f(0)$ using a half-normal detection function (and a gamma prior), where $f(0)$ is a quantity estimated from the distance data that allows observed counts to be adjusted for imperfect detection. The approach makes use of the conjugate property between the normal and gamma distributions. Other studies have built upon this approach. Eguchi and Gerrodette (2009) extended this model by including a binomial likelihood for the encounter rate along the line and described a joint posterior distribution for the density model and effective strip width. Gimenez et al. (2009) implemented an estimator for $f(0)$ using BUGS software, while Zhang (2011) developed an empirical Bayes estimator for $f(0)$. These studies follow a similar approach in that they present their methods for line-transect data and use the half-normal detection function. We describe a Bayesian approach to density estimation from distance sampling data for both line and point transect data applicable to any detection function that uses a hierarchical modeling approach.

Hierarchical distance sampling models have also been developed by e.g. Royle and Dorazio (2008, ch.7.1). These authors employ a likelihood that combines the detection function and the binomial model using the marginal probability of encounter (estimated from the detection function) and a data augmentation approach for unobserved groups. The data augmentation approach was adopted by Schmidt et al. (2012) who added a group size model and Conn et al. (2012) who extended the 
approach for double observer data.

In contrast, we use an integrated likelihood that combines the likelihood components of the detection and count models. For the latter, we use a Poisson likelihood for the distance sampling counts that incorporates a component corresponding to the detection function, thus allowing for undetected animals on the surveyed strip (line transects) or circular plot (point transects). In comparison to Eguchi and Gerrodette (2009) who use a binomial likelihood to scale up from density at the line to density in the study area, our Poisson model relates animal counts to covariates via a log-link function. This approach does not rely on random placement of samplers in the study area to the same extent as the design-based approach for the binomial model (Hedley and Buckland, 2004). Similar to Chelgren et al. (2011), we include a random effect for site in the Poisson model to accommodate correlated counts due to e.g. repeat counts at the same site. The parameter space is explored using a Metropolis-Hastings (MH) updating algorithm so that different prior distributions for the parameters are easily implemented, and a reversible jump Markov chain Monte Carlo (RJMCMC) algorithm allows for model uncertainty to be incorporated. This may include different key functions for the detection function model and different covariate combinations for both the detection function and the count models.

These developments were motivated by a large-scale experimental study to assess the effects of establishing conservation buffers along field margins on density of several species of conservation interest such as the northern bobwhite (Colinus virginianus). Pairs of points were set up at the edge of fields in farmland in 13 states in the USA. These pairs of points consisted of one point on a buffered treatment field and one on a nearby non-buffered control field and will be referred to as sites in the following. Point transect surveys of coveys (fall-winter stable social units of 10-15 individual birds) were conducted at least once but up to three times per year in autumn from 2006 to 2008 .

In the following we begin by developing the integrated likelihood (section 2), describe the Bayesian approach (section 3) and analyse bobwhite covey data using our Bayesian approach (and a maximum likelihood approach for comparison) (section 4). Lastly we contrast our Bayesian approach 
with existing studies using distance sampling likelihoods (section 5).

\section{An Integrated Likelihood for Distance Sampling Data}

To obtain abundance estimates of a population of interest using distance sampling methods, lines or points may be placed in the study area according to some design (see Buckland et al., 2001, for details). Each line or point is surveyed at least once following the distance sampling protocol where the observer travels along the line (line transects) or remains at the point for a fixed amount of time (point transects). Detections are recorded along with the perpendicular distance from the line to the detection or radial distance from the point to the detection. These distances may be recorded exactly or in predetermined distance intervals. Thus, surveys of this type produce two types of data: firstly, the observed distances $y_{e}$ with $e=1,2,3, \ldots, n$ ( $n$ being the total number of detections) or observed distances $n_{i}$ in each of $i=1,2,3, \ldots, I$ distance intervals (where $\sum_{i=1}^{I} n_{i}=n$ ); secondly, the observed number of detections or counts $n_{p}$ at line or point $p$ along with the effort data which at bare minimum consists of the size of the search area. In case detections are made of single animals, the observed counts at the line (point) are equivalent to the number of detections at the line (point). These two types of data, distances and counts, give rise to the two components of the integrated likelihood described in this section. However, if detections are made of groups of animals (rather than single individuals), a third type of data generated from a distance sampling survey is cluster size $s_{e}$ which represents the number of individuals within the eth detected group. For simplicity, we ignore cluster sizes for this study. Methods could, however, be extended to accomodate group sizes larger than one. This may be done by considering counts of individuals (rather than detections) in the count model described below or by including a model for cluster sizes. The latter may be desirable e.g. if group size data are overdispersed (e.g. Cañadas and Hammond, 2006; Schmidt et al., 2012).

In contrast to many existing covariate models for distance sampling data (e.g. Hedley and Buckland, 2004; Buckland et al., 2009), the proposed integrated likelihood deals with both components 
of the data simultaneously - similar to e.g. Royle et al. (2004) and Sillett et al. (2012). It consists of the likelihood components for the detection function, which is denoted by $L_{y}(\boldsymbol{\theta})$ for exact distance data (see eqn (5) below for interval data), and the Poisson likelihood for observed counts, $L_{n}(\boldsymbol{\beta} \mid \boldsymbol{\theta})$. We use $\boldsymbol{\theta}$ and $\boldsymbol{\beta}$ to summarise the detection function and Poisson model parameters, respectively. These are defined in more detail below. The integrated likelihood is the product of the two components:

$$
L_{n, y}(\boldsymbol{\beta}, \boldsymbol{\theta})=L_{y}(\boldsymbol{\theta}) L_{n}(\boldsymbol{\beta} \mid \boldsymbol{\theta})
$$

(modified from Buckland et al., 2004, ch. 2). We consider each individual likelihood component in $L_{n, y}(\boldsymbol{\beta}, \boldsymbol{\theta})$ and begin with $L_{y}(\boldsymbol{\theta})$.

\subsection{Likelihood component for the detection function}

Let $f(y \mid \boldsymbol{\theta})$ denote the probability density function of observed distances which is given as:

$$
f(y \mid \boldsymbol{\theta})=\frac{\pi(y) g(y \mid \boldsymbol{\theta})}{\int_{0}^{w} \pi(y) g(y \mid \boldsymbol{\theta}) d y},
$$

where $y$ is the observed distance from the line (point) and $w$ is the truncation distance (i.e. the furthest distance from the line (point) included in the analysis, Thomas et al., 2010). $\pi(y)$ describes the expected distribution of animals with respect to the line $(\pi(y)=1 / w)$ or point $\left(\pi(y)=2 y / w^{2}\right)$. The detection function $g(y \mid \boldsymbol{\theta})$ may be modelled e.g. as half-normal $\left(g(y \mid \boldsymbol{\theta})=\exp \left(-y^{2} / 2 \sigma^{2}\right)\right.$, with $\boldsymbol{\theta}=\{\sigma\})$ or hazard-rate $\left(g(y \mid \boldsymbol{\theta})=1-\exp \left(-(y / \sigma)^{-\tau}\right)\right.$, with $\left.\boldsymbol{\theta}=\{\sigma, \tau\}\right)$. The likelihood component, which is conditional on the number of detections $n$, may be expressed as:

$$
L_{y}(\boldsymbol{\theta})=\prod_{e=1}^{n} f\left(y_{e} \mid \boldsymbol{\theta}\right),
$$

where $y_{e}$ refers to the $e$ th detection (Buckland et al., 2004).

When detections are recorded in distance intervals, let $f_{i}$ denote the probability that a detected animal is in the $i$ th interval which is delineated by the cutpoints $c_{i-1}$ and $c_{i}$ : 


$$
f_{i}(\boldsymbol{\theta})=\frac{\int_{c_{i-1}}^{c_{i}} f\left(y_{i} \mid \boldsymbol{\theta}\right) d y}{\int_{0}^{w} f\left(y_{i} \mid \boldsymbol{\theta}\right) d y}
$$

where the truncation distance, $w$ corresponds to the outermost cutpoint. Then, the multinomial likelihood $L_{y G}$ replaces $L_{y}$ in eqn (1) and may be expressed as:

$$
L_{y G}(\boldsymbol{\theta})=\left(\frac{n !}{\prod_{i=1}^{I} n_{i} !}\right) \prod_{i=1}^{I} f_{i}(\boldsymbol{\theta})^{n_{i}},
$$

where $n_{i}$ is the number of detected animals in the $i$ th interval.

Note that in eqns (3) and (5), detections from all sites are pooled in one detection function. For modelling heterogeneity using multiple covariate distance sampling (MCDS) methods, the scale parameter $\sigma$ of the half-normal or hazard-rate detection function is modelled as a function of covariates $\left(\sigma(\boldsymbol{z})=\delta_{0} \times \exp \left(\sum_{q=1}^{Q} z_{q} \delta_{q}\right)\right.$, where $\boldsymbol{\delta}_{\boldsymbol{q}}, q=0,1,2, \ldots, Q$ replace $\sigma$ in $\boldsymbol{\theta}$ ) (Marques and Buckland, 2003) and the $z_{q}$ represent the values of the $q$ th covariate.

\subsection{Likelihood component for the count model}

For the log-linear Poisson model, $L_{n}(\boldsymbol{\beta} \mid \boldsymbol{\theta})$ we begin by considering a study design which consists of multiple sites each containing one or more lines (points) that are surveyed at least once. If all sites contain only one line (point) and surveys are not repeated, each count may be considered independent under conditions described by Buckland et al. (2001). However, if sites contain clusters of lines (points) and/or sites were surveyed more than once, this assumption is violated. We deal with this by grouping counts from the same site and fitting a random effect coefficient for each site in the following count model (see below in this section).

Here, we consider counts $n_{j p r}$ at visit $r$ to line or point $p$ at site $j$ as a Poisson random variable with $E\left(n_{j p r}\right)=\lambda_{j p r}$. To adjust counts for imperfect detection out to distance $w, f(y \mid \boldsymbol{\theta})$ from eqn (2) is used to estimate the effective area $\nu(\boldsymbol{\theta})$ which is defined as the area beyond which as many animals 
are seen as are missed within (Buckland et al., 2001). Thus density $D_{j p r}$ may be expressed as:

$$
D_{j p r}=\lambda_{j p r} / \nu(\boldsymbol{\theta})
$$

For line transects, $\nu(\boldsymbol{\theta})=2 l_{p} \int_{0}^{w} g(y \mid \boldsymbol{\theta}) d y$, where $l_{p}$ is the length of the line surveyed; for point transects $\nu(\boldsymbol{\theta})=2 \pi \int_{0}^{w} y g(y \mid \boldsymbol{\theta}) d y$. These definitions for $\nu$ are given for the case where all detections are pooled in a global detection function. When modelling heterogeneity, e.g. using MCDS methods, the effective area may vary between lines (points) and the global $\nu$ becomes $\nu_{j p r}$. However, when replacing $D_{j p r}$ with a covariate model $\left(D_{j p r}=\exp \left(\beta_{0}+b_{j}+\sum_{k=1}^{K} x_{k j p r} \beta_{k}\right)\right)$ and rearranging eqn (6) we obtain a model for the expected counts $\lambda_{j p r}$ which is now a function of the density model parameters $\boldsymbol{\beta}$ and conditional on detection function parameters $\boldsymbol{\theta}$ :

$$
\lambda_{j p r}(\boldsymbol{\beta} \mid \boldsymbol{\theta})=\exp \left(\beta_{0}+b_{j}+\sum_{k=1}^{K} x_{k j p r} \beta_{k}+\ln (\nu(\boldsymbol{\theta}))\right) .
$$

Here $\beta_{0}$ is the intercept, $b_{j}$ the random effect for site $j\left(b_{j} \sim N\left(0, \sigma_{b}^{2}\right)\right), x_{k}$ the $k$ covariates, $x_{k j p r}$ the covariate values measured during visit $r$ to that line or point and $\beta_{k}$ the associated coefficients. Vector $\boldsymbol{\beta}=\left\{\beta_{0}, \beta_{1}, \beta_{2}, \ldots, \beta_{K}, \sigma_{b}\right\}$ denotes the parameters associated with the covariates affecting densities and the random effect standard deviation. Eqn (7) is given for the general case where lines or points that may produce correlated counts, due to closeness in space and/or due to repeated measurements at the same line (point), are grouped together as site $j$. The inclusion of a random effect for site accommodates covariances for these measurements. However, in cases where lines (points) follow a random survey design (Buckland et al., 2001) and each line (point) is surveyed only once, the random effect term may be omitted.

Using this model for $\lambda_{j p r}$, the likelihood for the count model (eqn 7), conditional on detection function parameters $\boldsymbol{\theta}$, may be expressed as:

$$
L_{n}(\boldsymbol{\beta} \mid \boldsymbol{\theta})=\prod_{j=1}^{J} \int_{-\infty}^{\infty}\left(\prod_{p=1}^{P_{j}} \prod_{r=1}^{R_{j}} \frac{\left(\lambda_{j p r}\right)^{n_{j p r}} \exp \left(-\lambda_{j p r}\right)}{n_{j p r} !} \times \frac{1}{\sqrt{2 \pi \sigma_{b}^{2}}} \exp \left(-\frac{1}{2 \sigma_{b}^{2}} b_{j}^{2}\right)\right) d b_{j}
$$


where $J$ refers to the total number of sites, and $P_{j}$ and $R_{j}$ refer to the total number of lines (points) at and visits to the $j$ th site, respectively. $L_{n}(\boldsymbol{\beta} \mid \boldsymbol{\theta})$ forms the second likelihood component in eqn (1). Note that in a maximum likelihood context the likelihood function including a random effect (for which normality is assumed) is generally formulated with an integral as shown in eqn (8) as the random effect is integrated out analytically (or by approximation) and the individual coefficients $b_{j}$ are not estimated (e.g. McCulloch and Searle, 2001). In the Bayesian context, the random effect is not integrated out analytically. Here, we use a data augmentation scheme where the individual coefficients $b_{j}$ are included as parameters (or auxiliary variables) within the model and updated within the MCMC algorithm (see below).

\section{The Bayesian Approach}

\subsection{Hierarchical Models}

Using a Bayesian approach, random effects models can be implemented using hierarchical models where the standard deviation of the random effect ( $\sigma_{b}$ from eqn (8)) is considered a random variable with a distribution rather than as a fixed value (Davison 2003). Individual random effects coefficients $\left(b_{j}\right.$ from eqns (7) and (8)) are fitted in the model and updated at each iteration of the chain in a Markov chain Monte Carlo (MCMC) algorithm.

Prior beliefs regarding the parameters, such as knowledge obtained from a previous study, may be included in the current study via the prior distribution. This may allow inference on model parameters in cases where too few data exist in the current study to obtain maximum likelihood estimates with great precision (e.g. Eguchi and Gerrodette, 2009).

\subsection{MCMC Algorithm}

An MCMC algorithm is used to explore the posterior distribution of the parameters given the data. Commonly used MCMC methods are the Gibbs sampler and Metropolis-Hastings (MH) update. 
We focus on the MH update (Hastings, 1970; Metropolis et al., 1953) as some of the likelihood functions that may be used to form the posterior conditional distributions of parameters are nonstandard (e.g. half-normal or hazard-rate detection function that may include a covariate model for the scale parameter). In particular, we use a random walk single-update $\mathrm{MH}$ algorithm with normal proposal density. The proposal variance for each parameter may be obtained via pilottuning (Gelman et al., 1996). See Appendix 6.1 for details on the MH algorithm.

\subsection{Model Selection: Reversible Jump MCMC}

To discriminate between competing models, we treat the model itself as a parameter and form the joint posterior distribution over both parameters and models. To explore this posterior distribution we implement an RJMCMC algorithm (Green, 1995) where each iteration involves two steps; step 1: update parameters given the current model using the $\mathrm{MH}$ algorithm (within model move) as described above in section 3.2; step 2: update the model using an RJ algorithm (between model move). Posterior model probabilities are estimated as the proportion of time the chain spent in a particular model after the burn-in.

For the RJ step, two main strategies may be followed. In cases where models differ only in the combination of the same set of covariates, a single RJ step may involve going through each covariate and proposing to delete or add it depending on whether it is in the current model or not. This involves generating a value for the new parameter from a proposal distribution (if we propose to add it) or setting it to zero (if we propose to delete it) and calculating the acceptance probability each time we propose to add or delete a parameter.

In those cases where all parameters of the newly proposed model change, one RJ step involves generating new values for all parameters of the new model and accepting or rejecting the new model based on the above acceptance probability. A proposed move from a half-normal detection function model to a hazard-rate model represents a simple example for this scenario. For further details of the RJMCMC algorithm see Appendix 6.2. 


\section{Case Study}

\subsection{The Data}

As part of a study to assess the potential benefits of herbaceous buffers around agricultural fields, Mississippi State University, Department of Wildlife, Fisheries, and Aquaculture set up a monitoring program using point transects in a number of Midwestern and Southeastern states in the US (Evans et al., 2013). Survey points located at the edge of the field were paired up: one point on a buffered treatment field and the other on a non-buffered control field of the same agricultural use and within $1-3 \mathrm{~km}$ of the treatment point. Each pair of points will be referred to as a site in the following. Repeat visits were made to each point during fall of three survey years (2006-2008), and each detected northern bobwhite covey was recorded along with their estimated radial distance to the point. To facilitate unbiased distance estimation, observers used satellite images of the point location and surroundings to mark each detected covey. As no estimates of covey cluster sizes were obtained, we model cluster densities (rather than densities of individuals).

Only those 11 among the 13 original states in our study were included in the analysis that contained more than 50 detections of coveys: Georgia, Iowa, Illinois, Indiana, Kentucky, Missouri, Mississippi, North Carolina, South Carolina, Tennessee and Texas. Within these states, 447 sites

were visited 1-3 times in each survey year. The number of sites per state ranged from 30 to 61 . After defining a truncation distance of 500m following recommendations of Buckland et al. (2001), the analysed data included a total of 2545 detections with associated distances that were observed during 2534 counts (number of counts by state: GA 190, IA 221, IL 162, IN 217, KY 218, MO 352, MS 236, NC 244, SC 250, TN 219, TX 225).

\subsection{The Bayesian Approach}

We used eqns (3) and (8) to form the integrated likelihood function as shown in (1). Potential covariates included in the models for $L_{y}(\boldsymbol{\theta})$ and $L_{n}(\boldsymbol{\beta} \mid \boldsymbol{\theta})$ were the factor covariates year (three levels: 2006, 2007, 2008), type (two levels: Control or Treatment plot), state (11 levels) and the numeric 
covariate Julian day which was centred around its mean before the analyses (for the $L_{n}(\boldsymbol{\beta} \mid \boldsymbol{\theta})$ model only as it did not reveal any influence on detection probabilities during preliminary analyses using Distance software, Thomas et al., 2010). See Evans et al. (2013) for ecological details on modelling data from this study. Hence, eight different covariate combinations were possible for the detection function while 16 were possible for the density model.

We assume that counts from the different points of the same sites were (positively) correlated in the same manner as repeat counts of the same point. Hence, we included one random effect coefficient per site in the model (as described in section 2.2) where the same coefficient applied to all repeat counts at either of the two points belonging to the same site.

Uniform priors were placed on all parameters $\boldsymbol{\theta}$ and $\boldsymbol{\beta}$ for which bounds were chosen in preliminary analyses (Table 1). Generally this involved adding/subtracting two times the standard errors from the maximum likelihood estimate of the full model; however, bounds were extended if a parameter value reached either of these. If the parameter had natural bounds, e.g. zero for the lower bound of the random effect standard deviation, these were adopted.

To make summary statistics of parameters directly comparable to the maximum likelihood approach (see section 4.3), the last covariate levels (in numerical or alphabetic order) of detection function parameters were absorbed in the intercept to follow the parameterisation of factor levels in Distance software. Likewise, the first levels were absorbed in the intercept for the count model to follow parameterisation of factor levels in the glmer function from the lme4 package in R. Preliminary investigation of the distance data indicated that the hazard-rate detection function provided a much better fit than the half-normal. Hence, we included eight different hazard-rate models as choices for the probability density function of observed distances $f(y \mid \boldsymbol{\theta})$ in $L_{y}(\boldsymbol{\theta})$ : one global (with no covariates) and seven multiple covariate models. For the global model, only the scale and the shape parameters required estimation (see section 2 for details). The multiple covariate models contained additional parameters as the scale parameter was modelled as a function of one, two or three of the covariates. For $L_{n}(\boldsymbol{\beta} \mid \boldsymbol{\theta}), \lambda_{j p r}$ from eqn (7) was modelled including a intercept and combinations of the four covariates as well as a random effect for site. In preliminary analyses, we 
investigated whether the count data were overdispersed. We fitted a glmm with a quasipoisson distribution using the full models for both detection and counts to calculate the offset. A quasipoisson glmm can be fitted using the gamm function of the $m g \mathrm{cv}$ package. The estimate of the dispersion parameter was 1.10. Hence, we assumed that Poisson was appropriate to use.

The chain was started without any covariates for the detection and count models. During a single RJ step of each iteration, each of the covariates was proposed to be added or deleted depending on whether it was in the current model or not. Values $u$ for the new parameters contained in the new model were drawn from parameter-specific proposal distributions shown in Table 2. These were initially defined as normal distributions with mean and standard deviation equal to the maximum likelihood estimates and standard errors from the full models, however, we adjusted means (by averaging estimates across different models for the respective parameters) and standard deviations during pilot-tuning to improve model mixing.

To move from e.g. a global hazard-rate model to a model including a covariate, the global scale parameter $\sigma$ was converted into $\delta_{0} \times \exp \left(z_{1} \delta_{1}\right)$ with $\sigma=\delta_{0}$ and $u=\delta_{1}$, where $\delta_{1}$ is the coefficient associated with covariate $z_{1}$. The bijective function in this case (as well as in all the other possible model moves) was the identity function similar to the example shown in Appendix 6.2. Therefore, the Jacobian $|J|$ (from eqn (12) in Appendix 6.2) equalled one. We assume that all models were equally likely a priori, hence the probability of moving to model $m$ conditional on the chain being in model $m^{\prime}, P\left(m \mid m^{\prime}\right)$ was equal to $P\left(m^{\prime} \mid m\right)$ and vice versa for all possible model moves and cancelled when calculating the acceptance probability (see eqn (12)).

Proposal distributions for the MH step were normal where the mean was the current value of the parameter and the standard deviation was parameter-specific. The RJ and MH step together completed one iteration. A total of 100000 iterations were carried out where the first 10000 were considered the burn-in period and ignored when obtaining model probabilities and summary statistics for parameters. Visual inspection of raw trace plots from different starting points for parameters suggested that convergence had been achieved within 10000 iterations.

We conducted a prior sensitivity analysis where the uniform priors were replaced with normal 
priors for all parameters. We also tested the robustness of our estimated model probabilities by starting the RJMCMC algorithm from the full detection and count models (as opposed to the intercept only models). Both analyses revealed nearly identical results as those presented in section 4.4. Hence, we were confident that our results had converged.

\subsection{The Classical Approach}

To compare the Bayesian approach with the classical, the data were analysed using the two-stage approach (Buckland et al., 2009), extended to include a random effect for site in the count model (Oedekoven et al., 2013). The first step included fitting a detection function to observed distances by maximising the likelihood in eqn (3). The same eight hazard-rate models were explored as in the Bayesian approach, i.e. global and MCDS models with combinations of the covariates state, type and year. The effective area $\nu_{j p r}$ was estimated using the best model for $f(y \mid \boldsymbol{\theta})$.

In a second step, the effective area was incorporated into the count model for $\lambda_{j p r}$ and parameter estimates obtained using the glmer function of the lme4 package (Bates, 2009b) in R. The likelihood for the glmer function is equivalent to eqn (8), except that the random effect is integrated out using an approximation for the integral (Bates, 2009a). The same 16 models were explored as in the Bayesian approach including a fixed intercept and a random effect for site and combinations of the four covariates state, type, year and Julian day.

Best fitting models for both steps were found by minimum AIC values. As the effective area represents an estimate but is included in the model as if it was a known constant, non-parametric bootstrapping was used to estimate uncertainty (bootstrap standard errors (BSE) and 95\% confidence intervals) of parameter estimates. To implement a non-parametric bootstrap routine with 999 repeats, an automatic model selection was set up in $\mathrm{R}$ that included calls to the MCDS engine from the Distance software (Thomas et al., 2010) for the first step. For each bootstrap iteration, sites were resampled with replacement until the original number of sites was obtained (Buckland et al., 2009). To include model uncertainty in inference, the strategy followed was to select best fitting models based on minimum AIC values for each bootstrap iteration (Buckland et al., 1997). 


\subsection{Results}

In the following we refer to those models with highest probabilities as the preferred models. For the Bayesian approach, the preferred detection function model included the covariates year, type and state in the model for the scale parameter of the hazard-rate key function (probability $=1.00$ to two decimal places, Table 3). Two other models were visited within the RJMCMC algorithm with probabilities of $<0.001$ that included two (type and state) or one covariate only (type). The same model with all three covariates was the preferred model for the two-stage approach having been selected by AIC in $81 \%$ of bootstrap resamples. Three other models were selected: one with covariates year and state (16\%), one with type and state (2\%) and one with state alone (1\%).

For the count model, two models dominated the RJMCMC algorithm, the model with covariates type, Julian day and state as the preferred model (0.89 probability) and the full model (year + type + Julian day + state, 0.11 probability, Table 3). For the bootstrap the latter was the preferred model, selected in $89 \%$ of resamples, while the former was the second most frequently chosen model (10\%). Two other models were chosen during the bootstrap including the covariates year, type and state $(1 \%)$ and the model including covariates type and state $(<1 \%)$. Hence, the largest discrepancy in model probabilities between the two analysis methods was with regard to covariate year for which the total probabilities to be included in any model was 0.11 for the RJMCMC algorithm and 0.90 for the bootstrap (Table 3). However, 95\% confidence intervals obtained from the bootstrap overlapped zero for both year coefficients (Table 4) indicating that this covariate might have less importance than suggested by model probabilities for the bootstrap.

For the parameters of the detection function model, the posterior means of the parameters in the preferred model were in most cases similar to the maximum likelihood estimates resulting from the two-stage analysis of the original data (Table 4). The intercept for the scale parameter and the shape parameter were larger for the Bayesian approach while the coefficients for the scale parameter were on average smaller. Histograms of detections by state and estimated expected probability density functions (pdf) of observed distances from the Bayesian approach are shown in Figure 1. States SC and TX had the lowest average detection probabilities as indicated by the smallest coef- 
ficients for these states (Table 4). For these states, the steep decline after peak values for the pdf indicated rapidly declining detection probabilities beyond $\sim 100 \mathrm{~m}$ (Figure 1).

Interestingly, measures of uncertainty were mostly smaller for the Bayesian approach despite the fact that both stages from the two-stage approach were combined in one. The posterior standard deviations were smaller than the bootstrap standard errors for all detection function parameters. $95 \%$ credible intervals were narrower than the $95 \%$ confidence intervals for all but four detection function parameters (state coefficients IN, MS, NC and TN). Intervals from the two approaches overlapped in all cases for the detection function parameters.

For the count model, means and intervals were again similar between the two approaches (Table 4). However, slight discrepancies in means for count model coefficients existed which might have been due to that the best model from the two-stage approach contained the additional covariate year and/or to Monte Carlo error. Further reasons are discussed in section 5. Also, measures of uncertainty were again mostly smaller for the Bayesian approach: standard deviations from the Bayesian approach were smaller for all covariates in the count model compared to BSEs. $95 \%$ credible intervals were narrower for all coefficients of the count model compared to $95 \%$ confidence intervals, except for the covariate Julian day where they were equal. $95 \%$ credible and confidence intervals overlapped for all count model parameters. The only covariate selected for the preferred count model for the two-stage approach that was not also in the preferred model for the Bayesian approach was year. $95 \%$ confidence intervals for both year coefficients included zero indicating that this covariate might have been negligible for the count model.

The parameter of interest in these models was the coefficient for the level Treatment of the type covariate in the count model. This was $0.62(\mathrm{SD}=0.07)$ and $0.63(\mathrm{BSE}=0.12)$ for the Bayesian and the two-stage approach, respectively, indicating an increase in covey densities on treatment plots by $85 \%(E[\exp ($ type coefficient $)]=1.85)$ or $88 \%(\exp (0.63)=1.88)$ by the respective methods. As described above, expected densities can be calculated using the values of the $\boldsymbol{\beta}$ parameters from the count model. These values are obtained from the posterior distribution of these parameters for the Bayesian approach or maximum likelihood estimates for the two-stage approach (see above 
eqns (6) and (7) for details). For calculating baseline estimates of the expected covey densities for the RJMCMC algorithm and two-stage approach, we used those iterations from the respective methods where the preferred count model was chosen, excluding the burn-in iterations for the RJMCMC. Using eqn (7), we set the covariates to those levels that were absorbed by the intercept of the density model, i.e. year $=2006$, type $=$ Control, Julian day $=0$ (which is equivalent to its mean as we centered the data for this covariate) and state $=$ GA and added a random effect contribution $\left(0.5 \times \sigma_{b}^{2}\right.$ replaces $b_{j}$ from eqn (7) when calculating the average expected density across all sites). The estimated baseline expected density from the RJMCMC algorithm was 2.91 coveys per $\mathrm{km}^{2}(\mathrm{SD}=0.55,95 \% \mathrm{CRI}=(2.05,4.14))$. The posterior distribution of the expected density was right-skewed with a mode of 2.60 coveys per $\mathrm{km}^{2}$. The estimated expected density resulting from the two-stage approach was 2.44 coveys per $\mathrm{km}^{2}(\mathrm{BSE}=0.88,95 \% \mathrm{CI}=(1.25,4.66))$. Estimated expected densities for each state and type combination are shown in Figure 2.

\section{Discussion}

There are two main aspects in this paper that are innovative and deserve comparison to existing methods. We present a novel approach for combining the likelihood functions for analysing distance sampling data in section 2 which is easily applicable to both interval and exact distance data. We also present a Bayesian approach for analysing distance sampling data of multiple types in a straightforward manner. Different detection functions (the half-normal, hazard-rate or others) may easily be implemented. It may also be extended to include adjustment terms (added to the half-normal or hazard-rate model, Buckland et al. (2001)) or covariates in the shape parameter. We provide the $\mathrm{R}$ code as online supplementary material which is annotated for easy adaptation.

Bayesian methods have been used before for analysing line transect data with a global half-normal detection function (e.g. Royle and Dorazio, 2008, ch. 7.1; Eguchi and Gerrodette, 2009; Gimenez et al., 2009; Zhang, 2011), a half-normal with covariates (e.g. Gerrodette and Eguchi, 2011; Moore and Barlow, 2011) or a hazard-rate with one covariate (Schmidt et al., 2012). Our approach allows 
simultaneous exploration of model and parameter space including different detection functions and different covariate combinations for both the detection and count models via an RJMCMC algorithm. Conn et al. (2012) described an RJMCMC algorithm for distance sampling data. However, in their case, the RJ step refers to adding/deleting unobserved animals as part of the data augmentation and not to exploring different models for the detection function or counts.

The log-linear Poisson model for counts described in section 2 does not depend on a random survey design in contrast with the classical distance sampling approach and data arising from surveys conducted from platforms of opportunity may be used (Hedley and Buckland, 2004). It allows identification of relationships between abundance or density and parameters of interest, such as the type covariate in our case study, which may be of interest for designed experiments and wildlife management studies (see also Gerrodette and Eguchi, 2011). Similar to Hedley and Buckland (2004) and Buckland et al. (2009), our count model may be extended to include smooth functions for continuous covariates, e.g. by fitting polynomial splines using the B-spline basis, or the Poisson likelihood may be replaced with a negative binomial likelihood if more appropriate, e.g. in case overdispersion in the count data is present (e.g. Royle et al., 2004, Sillett et al., 2012). Our approach is similary to Hedley and Buckland (2004) in that we use the conditional formulation of the probability density function of observed distances (Buckland et al., 2001) and information on detection probabilities enters the count model via an offset; we model counts as density $\times$ effective area. However, Hedley and Buckland (2004) or Buckland et al. (2009) analyse their data in two stages. In their second stage count model, they condition on the estimate of the effective area derived from the first stage detection model. This requires conducting non-parametric bootstrapping so that uncertainty associated with estimating the detection function (and the effective area) propagates into the second stage count model. Our integrated likelihood approach estimates all parameters simultaneously allowing direct quantification of the precision of the parameters in the count model with taking proper account of the estimation of detection function parameters. Using the Poisson model with a random effect for estimating densities as defined in eqn (7) also allows us to accommodate correlated measurements due to closeness in space and/or time, as occurs 
e.g. when there are repeat counts at the same line (point). This differs from the integrated likelihood described by Royle et al. (2004). These authors considered the true but unknown abundances at the site as a random effect with a Poisson distribution (in their notation $N_{i} \sim \operatorname{Poisson}\left(\lambda_{i}\right)$ ) and integrate it out by summation. They derive a Poisson likelihood for the observed counts with expected value equal to $\lambda_{i} \pi_{k}(\theta)$, where $\pi_{k}(\theta)$ is obtained using the unconditional probability density function of observed distances and describes the probability that an animal occurs and is detected in the $k$ th distance interval. Hence, these authors model counts as abundance $N \times$ detected proportion of $N$ for each interval. Note that we use the term integrated likelihood in the spirit of integrating the likelihood components pertaining to two different data types (described in section 2), while Royle et al. (2004) use the same term in the context of integrating out a nuisance parameter (although they combine the likelihood components from two different data sources as well). In contrast to Royle et al. (2004), we model variations in observed counts between the different sites (those variations not explained by any of the fixed effects included in the model) as a normally distributed random effect with mean zero, hence accounting for correlations between measurements at the same sites. This represents an extension to Royle et al. who include one count per site in the analysis. With the inclusion of site random effects, our approach allows us to incorporate repeat counts from the same sites in the analysis. Our approach assumes that all counts from the same site are positively correlated and only requires estimation of one additional parameter, the random effect standard deviation. Chelgren et al. (2011) on the other hand, extended the approach of Royle et al. (2004) by including a random effect for plot by week in the abundance model which requires estimation of week-specific variance parameters. However, including random effects allows inference on the wider area that these sites represent and to obtain unbiased estimates of coefficients retained in the count model. Bias in coefficient estimates may occur for example if some sites with high bird densities were visited more frequently than those with low densities and this variation was not modelled as a fixed or random effect.

The comparison of summary statistics for model parameters from the Bayesian approach with those from the two-stage approach revealed some differences in means and point estimates (Table 
4) which cannot be due to prior sensitivity as we used uniform priors on all parameters for the Bayesian approach. We assume these differences may have been due to the fact that - as opposed to the two-stage approach - the likelihoods for both components of our model were combined for the integrated likelihood and influence each other. We argue, in concurrence with Johnson et al. (2010), that simultaneous estimation of all parameters in one stage represents a more realistic model without having to rely on the assumption of a true detection function model. Whether the smaller uncertainty estimates from the Bayesian approach compared to the two-stage approach were specific to our case study or can be expected in general is beyond the scope of this paper.

Our Bayesian approach provides improvements over previous approaches. Besides the often stated benefits for Bayesian analyses, e.g. allowing for prior information to be included, our Bayesian approach provided a particular benefit for using the integrated likelihood defined in section 2: it might be challenging in some cases, such as our case study, to find the maximum likelihood estimates for all parameters in one step. The covey data included a total of 2545 observed distances during 2534 counts and the full model included 31 parameters with a random effect (447 sites). Using maximum likelihood methods, the random effect coefficients are not estimated individually but are integrated out during the optimisation of the likelihood. However, due to the integrated nature of the detection and density models, functions such as glmer from the lme4 package in $\mathrm{R}$ may not be used as these treat the offset as a constant. Using the hierarchical model set up for the Bayesian approach, the random effect coefficients are included in the model specification and updated during each iteration. Due to this data augmentation method, no numerical integration is necessary providing a straightforward technique to explore the parameter space.

Bayesian methods also offer efficient exploration of model space with the use of RJMCMC. By contrast, using a maximum likelihood approach, a model selection routine that considered all possible model combinations for our case study would have required maximising $L_{y, n}(\boldsymbol{\beta}, \boldsymbol{\theta})$ for 128 models (possible combinations of eight detection functions and 16 count models). RJMCMC, on the other hand, allows incorporating model uncertainty into a single chain. 


\section{Acknowledgements}

The National CP-33 Monitoring Program was funded by the Multistate Conservation Grant Program (Grant MS M-1-T), which is supported by the Wildlife and Sport Fish Restoration Program and managed by the Association of Fish and Wildlife Agencies and US Fish and Wildlife Service. Further support was provided by the US Department of Agriculture (USDA) Farm Service Agency and USDA Natural Resources Conservation Service Conservation Effects Assessment Project. Collaborators included the AR Game and Fish Commission, GA Department of Natural Resources (DNR), IL DNR/Ballard Nature Center, IN DNR, IA DNR, KY Department of Fish and Wildlife Resources/KY Chapter of The Wildlife Society, MS Department of Wildlife, Fisheries and Parks, MO Department of Conservation, NE Game and Parks Commission, NC Wildlife Resources Commission, OH DNR, SC DNR, TN Wildlife Resources Agency, TX Parks and Wildlife Department, Southeast Quail Study Group and Southeast Partners In Flight. Cornelia S. Oedekoven was supported by a studentship jointly funded by the University of St Andrews and EPSRC, through the National Centre for Statistical Ecology. Some of the R functions for the two-stage approach were provided by Len Thomas.

\section{References}

Bates, D. (2009a). Adaptive Gauss-Hermite Quadrature for Generalized Linear or Nonlinear Mixed Models. R package version 0.999375-31. Technical report, http//lme4.r-forge.rproject.org/.

Bates, D. (2009b). Computational methods for mixed models. R package version 0.999375-31. Technical report, http//lme4.r-forge.r-project.org/.

Buckland, S. T., Anderson, D. R., Burnham, K. P., Laake, J. L., Borchers, D. L., and Thomas, L. (2001). Introduction to Distance Sampling. Oxford University Press. 
Buckland, S. T., Anderson, D. R., Burnham, K. P., Laake, J. L., Borchers, D. L., and Thomas, L. (2004). Advanced Distance Sampling. Oxford University Press.

Buckland, S. T., Burnham, K. P., and Augustin, N. H. (1997). Model selection: An integral part of inference. Biometrics 52(2), 603-618.

Buckland, S. T., Goudie, I. B. J., and Borchers, D. L. (2000). Wildlife population assessment: past developments and future directions. Biometrics 56, 1-12.

Buckland, S. T., Russell, R. E., Dickson, B. G., Saab, V. A., Gorman, D. G., and Block, W. M. (2009). Analysing designed experiments in distance sampling. Journal of Agricultural, Biological and Environmental Statistics 14, 432-442.

Cañadas, A. and Hammond, P. S. (2006). Model-based abundance estimates for bottlenose dolphins off southern Spain: implications for conservation and management. Journal of Cetacean Research and Management 8(1), 13-27.

Chelgren, N. D., Samora, B., Adams, M. J., and McCreary, B. (2011). Using spatiotemporal models and distance sampling to map the space use and abundance of newly metamorphosed Western toads (Anaxyrus boreas). Herpetological Conservation and Biology 6(2), 175-190.

Conn, P. B., Laake, J. L., and Johnson, D. S. (2012). A Hierarchical Modeling Framework for Multiple Observer Transect Surveys. PLoS ONE 7(8), e42294.

Davison, A. C. (2003). Statistical Models. Cambridge University Press.

Durban, J. and Elston, D. (2005). Mark-recapture with occasion and individual effects: Abundance estimation through Bayesian model selection in a fixed dimensional parameter space. Journal of Agricultural, Biological, and Environmental Statistics 10, 291-305.

Eguchi, T. and Gerrodette, T. (2009). A Bayesian approach to line-transect analysis for estimating abundance. Ecological Modelling 220, 1620-1630. 
Evans, K. O., Burger, L. W., Oedekoven, C. S., Smith, M. D., Riffell, S. K., Martin, J. A., and Buckland, S. T. (2013). Multi-region response to conservation buffers targeted for northern bobwhite. The Journal of Wildlife Management 77, 716-725.

Gelman, A., Roberts, G. O., and Gilks, W. R. (1996). Bayesian statistics, chapter Efficient Metropolis jumping rules, pages 599-608. Oxford University Press, Oxford.

Gerrodette, T. and Eguchi, T. (2011). Precautionary design of a marine protected area based on a habitat model. Endangered Species Research 15(2), 159-166.

Gimenez, O., Bonner, S. J., King, R., Parker, R. A., Brooks, S. P., Jamieson, L. E., Grosbois, V., Morgan, B. J., and Thomas, L. (2009). WinBUGS for population ecologists: Bayesian modeling using Markov chain Monte Carlo Methods. In Thomson, D. L., Cooch, E. G., and Conroy, M. J., editors, Modeling Demographic Processes In Marked Populations, volume 3 of Environmental and Ecological Statistics, pages 883-915. Springer US.

Green, P. J. (1995). Reversible jump Markov chain Monte Carlo computation and Bayesian model determination. Biometrika 82(4), 711-732.

Hastings, W. K. (1970). Monte Carlo sampling methods using Markov chains and their applications. Biometrika 57(1), 97-109.

Hedley, S. L. and Buckland, S. T. (2004). Spatial models for line transect sampling. Journal of Agricultural, Biological and Environmental Statistics 9, 181-199.

Johnson, D. S., Laake, J. L., and Ver Hoef, J. M. (2010). A model-based approach for making ecological inference from distance sampling data. Biometrics 66, 310-318.

Karunamuni, R. J. and Quinn, T. J. (1995). Bayesian estimation of animal abundance for line transect sampling. Biometrics 51, 1325-1337.

King, R., Morgan, J., Gimenez, O., and Brooks, S. (2010). Bayesian Analysis for Population Ecology. Chapman \& Hall/CRC. 
Marcot, B. G., Holthausen, R. S., Raphael, M. G., Rowland, M. M., and Wisdom, M. J. (2001). Using Bayesian belief networks to evaluate fish and wildlife population viability under land management alternatives from an environmental impact statement. Forest Ecology and Management 153, 29-42.

Marques, F. F. C. and Buckland, S. T. (2003). Incorporating covariates into standard line transect analyses. Biometrics 53, 924-935.

McCulloch, E. C. and Searle, S. R. (2001). Generalized, Linear, and Mixed Models. John Wiley \& Sons, Inc.

Metropolis, N., Rosenbluth, A. W., Rosenbluth, M. N., Teller, A. H., and Teller, E. (1953). Equations of state calculations by fast computing machines. Journal of Chemical Physics 21, 10871091.

Moore, J. E. and Barlow, J. (2011). Bayesian state-space model of fin whale abundance trends from a 1991-2008 time series of line-transect surveys in the California Current. Journal of Applied Ecology 48, 1195-1205.

Oedekoven, C. S., Buckland, S. T., Mackenzie, M. L., Evans, K. O., and Burger, L. W. (2013). Improving distance sampling: accounting for covariates and non-independency between sampled sites. Journal of Applied Ecology 50(3), 786-793.

Royle, A. and Dorazio, R. M. (2008). Hierarchical Modeling and Inference in Ecology: The Analysis of Data from Populations, Metapopulations and Communities. Academic Press, San Diego, CA.

Royle, J. A., Dawson, D. K., and Bates, S. (2004). Modelling abundance effects in distance sampling. Ecology 85(6), 1591-1597.

Schmidt, J. H., Lindberg, M. S., Johnson, D. S., Conant, B., and King, J. (2009). Evidence of 
Alaskan trumpeter swan population growth using Bayesian hierarchical models. The Journal of Wildlife Management 73(5), 720-727.

Schmidt, J. H., Rattenbury, K. L., Lawler, J. P., and MacCluskie, M. C. (2012). Using distance sampling and hierarchical models to improve estimates of Dall's sheep abundance. The Journal of Wildlife Management 76(2), 317-327.

Sillett, T. S., Chandler, R. B., Royle, J. A., Kéry, M., and Morrison, S. A. (2012). Hierarchical distance-sampling models to estimate population size and habitat-specific abundance of an island endemic. Ecological Applications 22, 1997-2006.

Thomas, L., Buckland, S. T., Rexstad, E. A., Laake, J. L., Strindberg, S., Hedley, S. L., Bishop, J. R. B., Marques, T. A., and Burnham, K. P. (2010). Distance software: design and analysis of distance sampling surveys for estimating population size. Journal of Applied Ecology 47, 5-14.

Zhang, S. (2011). On parametric estimation of population abundance for line transect sampling. Environmental and Ecological Statistics 18, 79-92.

\section{Appendix}

\subsection{Metropolis-Hastings}

We use a single-update random walk $\mathrm{MH}$ algorithm where we cycle through each parameter in $L_{n, y}(\boldsymbol{\beta}, \boldsymbol{\theta})$. To use a simple scenario, assume $\boldsymbol{\beta}=\left\{\beta_{0}, \sigma_{b}\right\}$. Then, e.g. for parameter $\beta_{0}$ with

current value $\beta_{0}^{t}$ we propose to move to a new state, $\beta_{0}^{\prime}$, with $\beta_{0}^{\prime} \sim\left(\beta_{0}^{t}, \sigma_{\beta_{0}}^{2}\right)$. This newly proposed state is accepted as the new state with probability $\alpha\left(\beta_{0}^{\prime} \mid \beta_{0}^{t}\right)$ given by:

$$
\alpha\left(\beta_{0}^{\prime} \mid \beta_{0}^{t}\right)=\min \left(1, \frac{L_{n, y}\left(\beta_{0}^{\prime}, \sigma_{b}^{t}, \boldsymbol{\theta}^{t}\right) p\left(\beta_{0}^{\prime}\right) q\left(\beta_{0}^{t} \mid \beta_{0}^{\prime}\right)}{L_{n, y}\left(\beta_{0}^{t}, \sigma_{b}^{t}, \boldsymbol{\theta}^{t}\right) p\left(\beta_{0}^{t}\right) q\left(\beta_{0}^{\prime} \mid \beta_{0}^{t}\right)}\right)
$$

Here, $q\left(\beta_{0}^{\prime} \mid \beta_{0}^{t}\right)$ denotes the proposal density of $\beta_{0}^{\prime}$ given the current state is $\beta_{0}^{t}$. We note that the terms $q\left(\beta_{0}^{t} \mid \beta_{0}^{\prime}\right)$ and $q\left(\beta_{0}^{\prime} \mid \beta_{0}^{t}\right)$ cancel in the acceptance probability since we use a symmetrical 
proposal distribution. The analogous MH updates are used for random effect coefficients. Proposal variances are chosen via pilot-tuning.

\subsection{Model selection: Reversible Jump MCMC}

The joint posterior distribution of models and parameters is given (up to proportionality) by:

$$
\pi_{n, y}\left(\boldsymbol{\beta}_{m}, \boldsymbol{\theta}_{m}, m\right) \propto L_{n, y}\left(\boldsymbol{\beta}_{m}, \boldsymbol{\theta}_{m}, m\right) p\left(\boldsymbol{\beta}_{m}, \boldsymbol{\theta}_{m} \mid m\right) p(m)
$$

where $L_{n, y}\left(\boldsymbol{\beta}_{m}, \boldsymbol{\theta}_{m}, m\right)$ denotes the probability density function of the data given current parameter values $\boldsymbol{\beta}_{m}$ and $\boldsymbol{\theta}_{m}$ and model $m, p\left(\boldsymbol{\beta}_{m}, \boldsymbol{\theta}_{m} \mid m\right)$ the prior distribution for model parameters $\boldsymbol{\beta}_{m}$ and $\boldsymbol{\theta}_{m}$ and $p(m)$ the prior probability of model $m$. The RJMCMC algorithm is used to explore the parameter and model space simultaneously (Green, 1995).

Each iteration involves two steps: a within model move and a between model move. During the within model move, the Metropolis-Hastings $(\mathrm{MH})$ algorithm is used to update the parameters given the model (as described above in section 3.2). During the between model move, the reversible jump (RJ) step, model $m$ conditional on the current parameter values is updated. This move involves a proposal to update the model itself; suppose the chain is in model $m$ and we propose to move to model $\mathrm{m}^{\prime}$. A bijective function describes the relationship between the current and proposed parameters and is used to convert parameters from model $m$ to parameters for model $m^{\prime}$. In a simple scenario, say, where model $m$ contains parameters $\boldsymbol{\beta}=\left\{\beta_{0}, \beta_{1}\right\}$ and model $m^{\prime}$ contains parameters $\boldsymbol{\beta}^{\prime}=\left\{\beta_{0}^{\prime}, \beta_{2}^{\prime}\right\}$, the bijective function might be expressed as an identity function:

$$
\beta_{0}^{\prime}=\beta_{0} \quad u^{\prime}=\beta_{1} \quad \beta_{2}^{\prime}=u .
$$

Here $u$ and $u^{\prime}$ are random samples from some proposal distributions for the respective parameters. The acceptance probability may then be expressed as:

$$
A=\frac{\pi_{n, y}\left(\boldsymbol{\beta}^{\prime}, m^{\prime}\right) P\left(m \mid m^{\prime}\right) q^{\prime}\left(u^{\prime}\right)}{\pi_{n, y}(\boldsymbol{\beta}, m) P\left(m^{\prime} \mid m\right) q(u)}|J|,
$$


where $P\left(m^{\prime} \mid m\right)$ denotes the probability of proposing to move to model $m^{\prime}$ given that the chain is in model $m, q(u)$ and $q^{\prime}\left(u^{\prime}\right)$ are the proposal densities of $u$ and $u^{\prime}$ and $|J|$ is the Jacobian (which equals one if the bijective function is the identity function). 
$\mathrm{GA}, \mathrm{n}=\mathbf{3 1 2}$

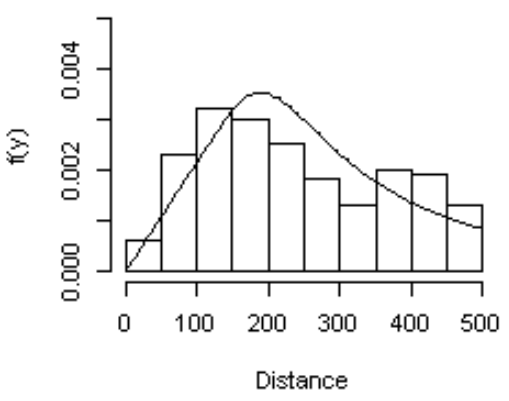

IN, $n=277$

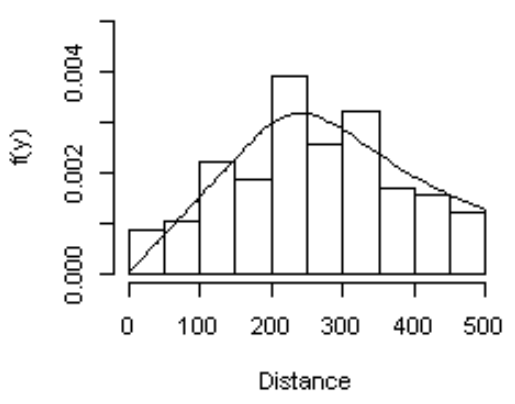

MS, $n=347$

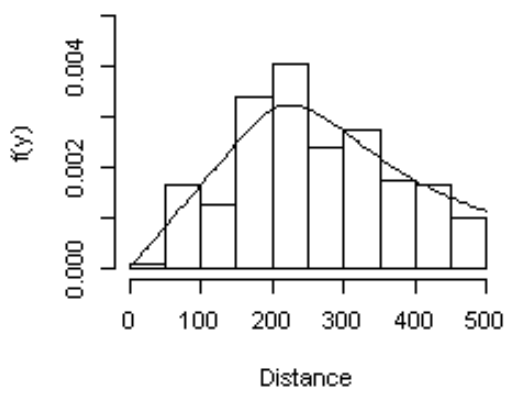

TN, $n=259$

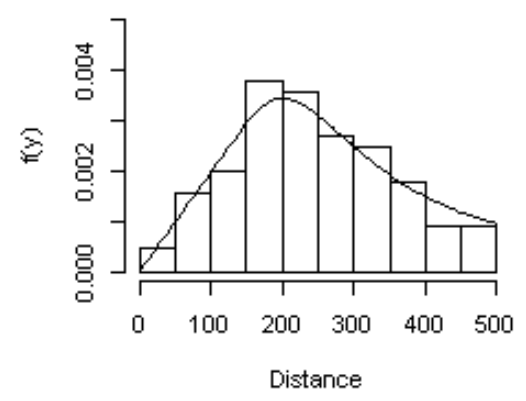

IA, $n=268$

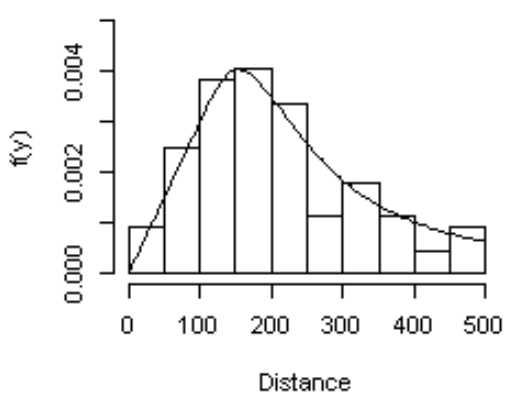

$K Y, n=307$

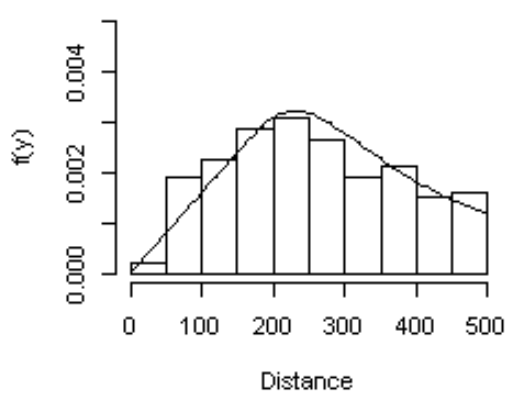

NC, $n=290$

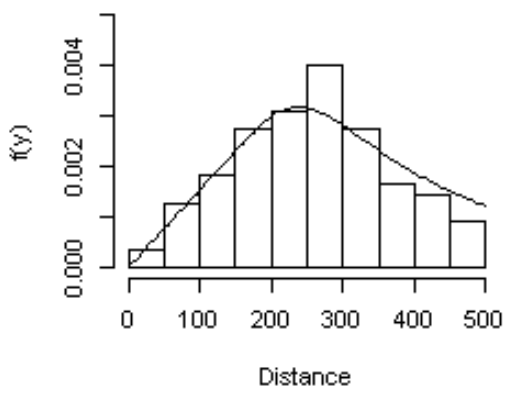

TX, $n=667$

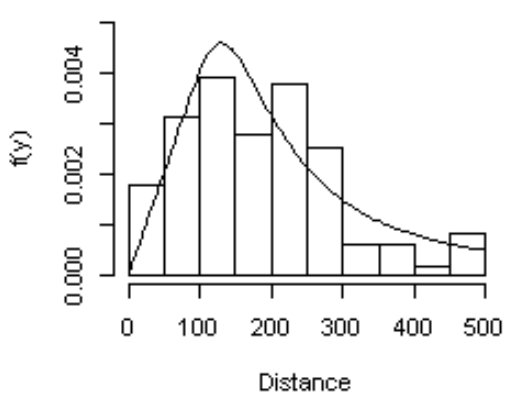

IL, $\mathbf{n}=239$

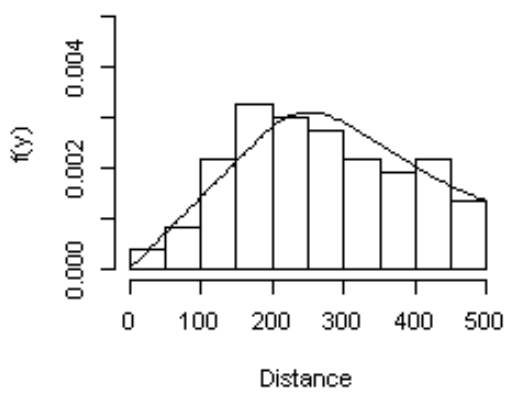

MO, $n=803$

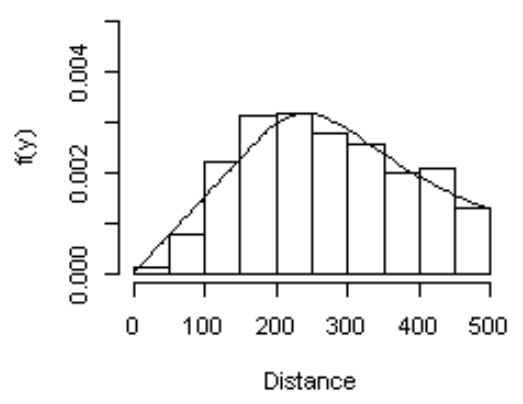

SC, $n=331$

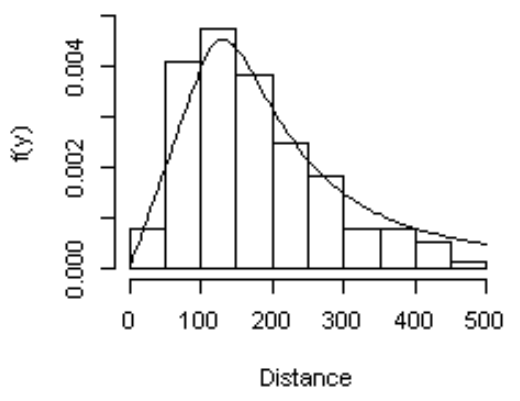

Figure 1: Estimated probability density functions, $f(y)$ (y-axis), using mean parameter values from the Bayesian approach (Table 4) against radial distance from the point (x-axis, in metres), and histograms of detections for each of 11 states (GA, IA, IL, IN, KY, MO, MS, NC, SC, TN, TX); here, $n$ represents the number of detections for the respective states. For each state, $f(y)$ was averaged over all levels of covariates year and type. 


\section{Estimated covey density}

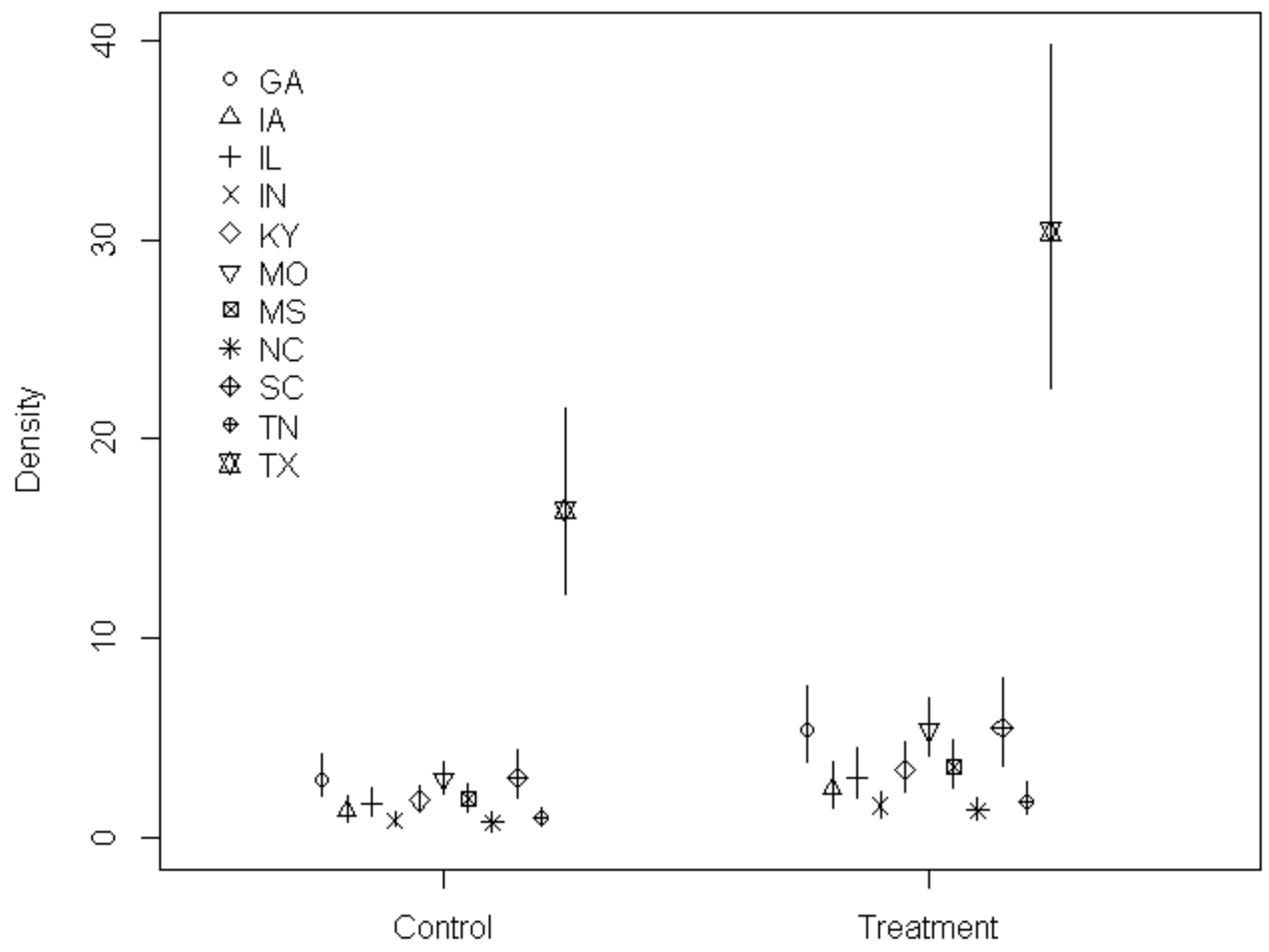

Field type

Figure 2: Estimated covey densities (coveys per $\mathrm{km}^{2}$ ) and $95 \%$ CRIs for each of 11 states shown for unbuffered control and buffered treatment fields. 
Table 1: Lower and upper bounds for uniform prior distributions for all model parameters. The different states included GA, IA, IL, IN, KY, MO, MS, NC, SC, TN and TX.

\begin{tabular}{l|cc}
\hline \hline Parameters & Lower & Upper \\
\hline Detection Model & 1 & 100000 \\
Scale Intercept: & 1 & 20 \\
Shape: & -3 & 3 \\
Year levels: 2006, 2007 & -2.5 & 2.5 \\
Type level: Control & -2.5 & 2.5 \\
State levels: GA:TN & & \\
Count Model & -20 & -7 \\
Intercept: & -1 & 1 \\
Year levels: 2007, 2008 & 0 & 1 \\
Type level: Treatment & -0.1 & 0.1 \\
Julian Day: & -3 & 3 \\
State level: IA:TX & 0 & 2 \\
Random effect standard deviation & & \\
\hline
\end{tabular}


Table 2: Mean and standard deviation (SD) of Normal proposal distributions for parameters proposed to be added or deleted during the RJ step of the RJMCMC algorithm. All parameters were categorical, except for continuous Julian day.

\begin{tabular}{l|cc}
\hline \hline Parameters & Mean & SD \\
\hline Detection Model & & \\
Year level 2006 & 0.11 & 0.10 \\
Year level 2007 & -0.15 & 0.10 \\
Type level: Control & 0.50 & 0.10 \\
State level: GA & 0.42 & 0.10 \\
State level: IA & 0.21 & 0.10 \\
State level: IL & 0.70 & 0.10 \\
State level: IN & 0.67 & 0.10 \\
State level: KY & 0.64 & 0.10 \\
State level: MO & 0.69 & 0.10 \\
State level: MS & 0.61 & 0.10 \\
State level: NC & 0.66 & 0.10 \\
State level: SC & 0.03 & 0.10 \\
State level: TN & 0.47 & 0.10 \\
Count Model & & \\
Year level: 2007 & 0.16 & 0.05 \\
Year level: 2008 & 0.08 & 0.05 \\
Type level: Treatment & 0.42 & 0.10 \\
Julian Day: & -0.01 & 0.01 \\
State level: IA & 0.71 & 0.24 \\
State level: IL & -0.49 & 0.24 \\
State level: IN & -1.16 & 0.23 \\
State level: KY & -0.41 & 0.22 \\
State level: MO & 0.01 & 0.20 \\
State level: MS & -0.38 & 0.22 \\
State level: NC & -1.36 & 0.23 \\
State level: SC & 0.07 & 0.22 \\
State level: TN & -1.05 & 0.23 \\
State level: TX & 1.77 & 0.21 \\
\hline
\end{tabular}


Table 3: Models and their probabilities resulting from RJMCMC and bootstrap analyses. Each density model included an intercept and a random effect for site in addition to shown covariates $(\mathrm{JD}=$ Julian day). Model probabilities refer to the percentage the respective models were chosen during 90000 iterations (after 10000 iterations of burn-in) for RJMCMC and during 999 bootstrap iterations.

\begin{tabular}{l|cc}
\hline \hline Model & RJMCMC & Two-Stage \\
\hline Detection Model & $<0.001$ & - \\
MCDS: Type & - & 0.01 \\
MCDS: State & - & 0.16 \\
MCDS: Year + State & $<0.001$ & 0.02 \\
MCDS: Type + State & 1.00 & 0.81 \\
MCDS: Year + Type + State & & \\
Count Model & - & $<0.005$ \\
Type + State & - & 0.01 \\
Year + Type + State & 0.89 & 0.10 \\
Type + JD + State & 0.11 & 0.89 \\
Year + Type + JD + State & & \\
\hline
\end{tabular}


Table 4: Mean, standard deviation (SD) and 95\% credible intervals (CRI) from the RJMCMC analysis along with maximum likelihood estimates (MLE), bootstrap standard errors (BSE) and 95\% confidence intervals (CI) using the two-stage approach for the models with the highest probabilities (see Table 3 for model probabilities). Units of measurements were metres for the detection function model and square metres for the count model.

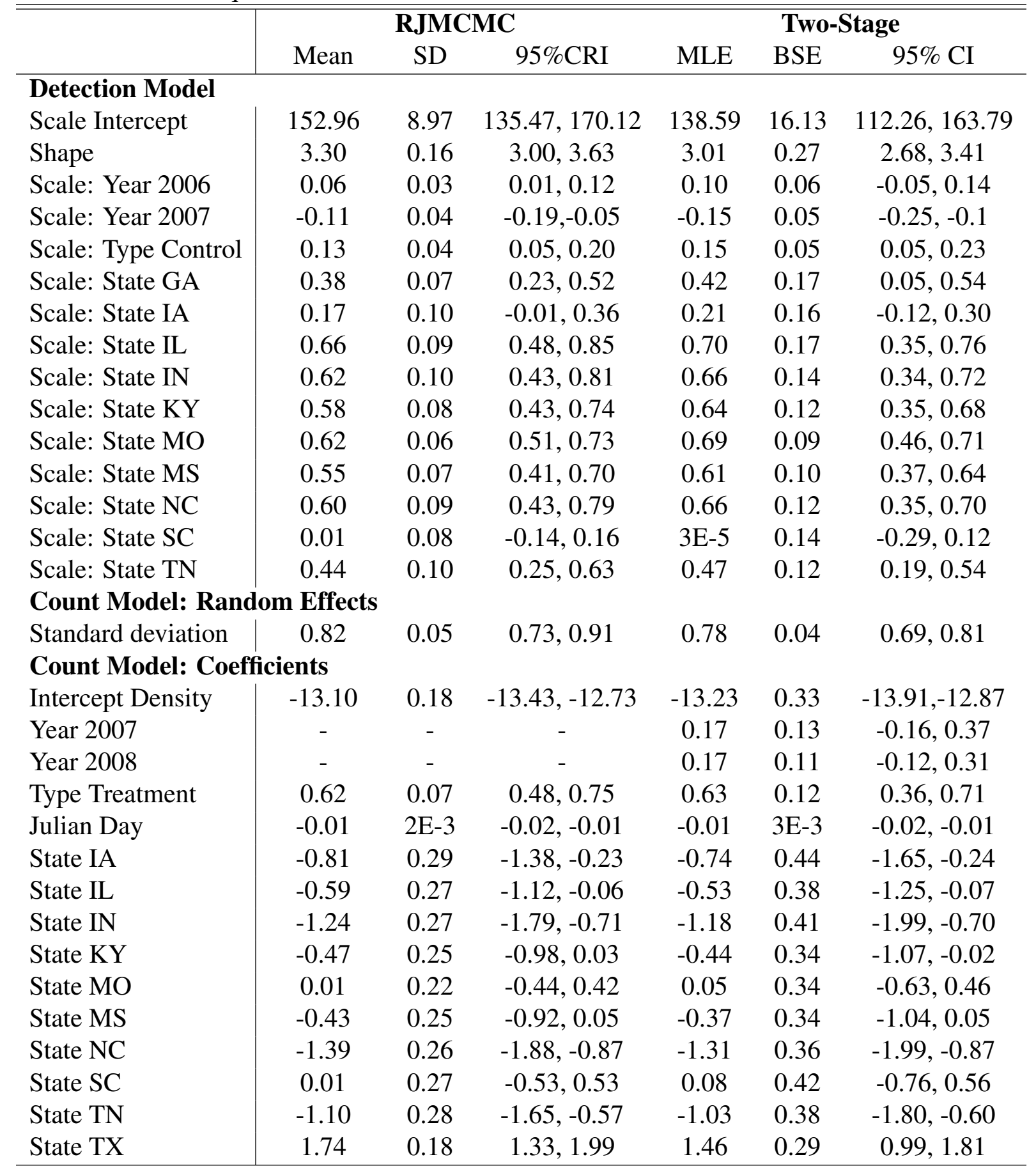

